\title{
On The Covering RADius OF CODES OVer $\mathbf{Z}_{6}$
}

\author{
P. Chella Pandian \\ Department of Mathematics Srimad Andavan Arts and Science College (Autonomous) \\ Tiruchirappalli - 620 005, Tamilnadu, India.
}

\begin{abstract}
In this correspondence, we give lower and upper bounds on the covering radius of codes over the finite ring $Z_{6}$ with respect to different distances such as Hamming, Lee, Euclidean and Chinese Euclidean. We also determine the covering radius of various Block Repetition Codes over $Z_{6}$.
\end{abstract}

\section{KEYWORDS}

Covering radius, Codes over finite rings, Hamming codes.(2010) Mathematical Subject Classification: 94B25, 94B05, 11H31, $11 \mathrm{H} 71$.

\section{INTRODUCTION}

In the last decade, there are many researchers doing research on code over finite rings. There has much interest in codes over finite rings in recent years, especially the rings $Z_{2 \mathrm{k}}$ where $2 \mathrm{k}$ denotes the ring of integers modulo $2 \mathrm{k}$. In particular, codes over $\mathrm{Z}_{4}$ have been widely studied $[1,2,3,4$, 5]. Good binary linear and non-linear codes can be obtained from codes over $Z_{4}$ via the gray map. The covering radius of binary linear codes was studied [6,7]. Recently the covering radius of codes over $\mathbf{Z}_{4}$ has been investigated with respect to Lee, Euclidean distances [1,10] and Chinese Euclidean distance [8]. In 1999, Sole et al gave many upper and lower bounds on the covering radius of a code over $\mathrm{Z}_{4}$ with different distances. In the recent paper, the covering radius of some codes over $Z_{6}$ have been investigated. In this correspondence, we consider the finite ring is the set $\mathrm{Z}_{6}$ of integers modulo 6 .

A linear code $\mathrm{C}$ of length $\mathrm{n}$ over $\mathrm{Z}_{6}$ is an additive subgroup of $Z_{6}^{n}$. An element of $\mathrm{C}$ is called a codeword of $\mathrm{C}$ and a generator matrix of $\mathrm{C}$ is a matrix whose rows generate $\mathrm{C}$. The Hamming weight $\mathrm{w}_{\mathrm{H}}(\mathrm{x})$ of a vector $\mathrm{x}$ in $Z_{6}^{n}$ is the number of non-zero coordinates.

In [11], the Lee weight for a codeword $\mathrm{x}=\left(\mathrm{x}_{1}, \mathrm{x}_{2}, \ldots, \mathrm{x}_{\mathrm{n}}\right) \in Z_{6}^{n}$ is defined by

$$
\mathrm{w}_{\mathrm{L}}(\mathrm{x})=\sum_{i=1}^{n}\left\{\left|x_{i} \| 6-x_{i}\right|\right\} \text {. }
$$

The Lee distance between the codeword's $\mathrm{x}$ and $\mathrm{y} \in Z_{6}^{n}$ is defined as $\mathrm{d}_{\mathrm{L}}(\mathrm{x}, \mathrm{y})=\mathrm{w}_{\mathrm{L}}(\mathrm{x}-\mathrm{y})$.

The Euclidean weight is given by the relation 


$$
\mathrm{w}_{\mathrm{E}}(\mathrm{x})=\sum_{i=1}^{n}\left\{\left|x_{i}\right|^{2}\left|6-x_{i}\right|^{2}\right\}
$$

and Euclidean distance between the codeword's $\mathrm{x}$ and $\mathrm{y} \in Z_{6}^{n}$ is defined as $\mathrm{d}_{\mathrm{E}}(\mathrm{x}, \mathrm{y})=\mathrm{w}_{\mathrm{E}}(\mathrm{x}-\mathrm{y})$.

The Chinese Euclidean weight $\mathrm{w}_{\mathrm{CE}}(\mathrm{x})$ of a vector $\mathrm{x} \in Z_{6}^{n}$ is

$$
\sum_{i=1}^{n}\left\{\left|2-2 \cos \left(\frac{2 \pi x_{i}}{6}\right)\right|\right\}
$$

and the Chinese Euclidean distance between the code words $\mathrm{x}$ and $\mathrm{y} \in Z_{6}^{n}$ is defined as $\mathrm{d}_{\mathrm{CE}}(\mathrm{x}, \mathrm{y})=\mathrm{w}_{\mathrm{CE}}(\mathrm{x}-\mathrm{y})$.

The Hamming, Lee, Euclidean and Chinese Euclidean distances $d_{H}(x, y), d_{L}(x, y), d_{E}(x, y)$ and $\mathrm{d}_{\mathrm{CE}}(\mathrm{x}, \mathrm{y})$ between two vectors $\mathrm{x}$ and $\mathrm{y}$ are $\mathrm{w}_{\mathrm{H}}(\mathrm{x}-\mathrm{y}), \mathrm{w}_{\mathrm{L}}(\mathrm{x}-\mathrm{y}), \mathrm{w}_{\mathrm{E}}(\mathrm{x}-\mathrm{y})$ and $\mathrm{w}_{\mathrm{CE}}(\mathrm{x}-\mathrm{y})$ respectively. The minimum Hamming, Lee, Euclidean and Chinese Euclidean weights $d_{H}, d_{L}, d_{E}$ and $d_{C E}$ of $C$ are the smallest Hamming, Lee, Euclidean and Chinese Euclidean weights among all non-zero codewords of $\mathrm{C}$ respectively.

A linear Gray map $\phi$ from $Z_{6}^{n} \rightarrow Z_{2}^{n} \times Z_{3}^{n}$ is the coordinates-wise extension of the function from $Z_{6}^{n}$ to $Z_{2}^{n} \times Z_{3}^{n}$ defined by $0 \rightarrow(0,0), 1 \rightarrow(1,1), 2 \rightarrow(0,2)$,

$3 \rightarrow(1,0), 4 \longrightarrow(0,1)$ and $5 \longrightarrow(1,2)$. The image $\phi$, of a linear code $\mathrm{C}$ over $\in Z_{6}^{n}$ of length $\mathrm{n}$ by the Gray map, is a mixed binary/ternary code of length $2 \mathrm{n}[11]$.

Two codes are said to be equivalent if one can be obtained from the other by permuting the coordinates or changing the signs of certain coordinates or multiplying non-zero element in a fixed column. Codes differing by only a permutation of coordinates are called permutationequivalent.

Any linear code $C$ over $Z_{6}$ is permutation-equivalent to a code with generator matrix $G$ (the rows of $\mathrm{G}$ generate $\mathrm{C}$ ) of the form

$$
G=\left[\begin{array}{cccc}
I_{k_{1}} & A_{1,2} & A_{1,3} & A_{1,4} \\
0 & 2 I_{k_{2}} & 2 A_{2,3} & 2 A_{2,4} \\
0 & 0 & 3 I_{k_{3}} & 3 A_{3,4}
\end{array}\right]
$$

Where $\mathrm{A}_{\{\mathrm{i}, j\}}$ are matrices with entries 0 or 1 for $\mathrm{i}>1$ and $\mathrm{I}_{\mathrm{k}}$ is the identity matrix of order $\mathrm{k}$. Such a code is said to have rank $\left\{1^{\mathrm{k} 1}, 2^{\mathrm{k} 2}, 3^{\mathrm{k} 3}\right.$ or simply rank $\left\{\mathrm{k}_{1}, \mathrm{k}_{2}, \mathrm{k}_{3}\right\}$ and $\mid \mathrm{Cl}=6^{\mathrm{k} 1} 3^{\mathrm{k} 2} 2^{\mathrm{k} 3}$. If $\mathrm{k}_{2}=\mathrm{k}_{3}=0$, then the rank of $\mathrm{C}$ is $\left\{\mathrm{k}_{1}, 0,0\right\}$ or simply $\mathrm{k}_{1}=\mathrm{k}$. 
In this paper, we define the covering radius of codes over $\mathrm{Z}_{6}$ with respect to different distances. Section 2 contains basic results for the covering radius of codes over $\mathrm{Z}_{6}$.

Determines the covering radius of different types of block repetition codes are given in Section 3.

\section{Covering Radius of Codes}

Let $\mathrm{d}$ be the general distance out of various possible distances such as Hamming, Lee, Euclidean and Chinese Euclidean. The covering radius of a code $\mathrm{C}$ over $\mathrm{Z}_{6}$ with respect to a general distances d is given by $r_{d}(C)=\max _{u \in z_{6}^{n}}\left\{\min _{c \in C}\{d(u, c)\}\right\}$

The following result of Mattson [6] is useful for computing covering radius of codes over rings generalized easily from codes over finite fields.

Proposition 2. 1. If $\mathrm{C}_{0}$ and $\mathrm{C}_{1}$ are codes over $\mathrm{Z}_{6}$ generated by matrices $\mathrm{G}_{0}$ and $\mathrm{G}_{1}$ respectively and if $\mathrm{C}$ is the code generated by $G=\left(\begin{array}{cc}0 & G_{1} \\ G_{0} & A\end{array}\right)$ then $\mathrm{r}_{\mathrm{d}}(\mathrm{C}) \leq \mathrm{r}_{\mathrm{d}}\left(\mathrm{C}_{0}\right)+\mathrm{r}_{\mathrm{d}}\left(\mathrm{C}_{1}\right)$ and the covering radius of $D$ (concatenation of $C_{0}$ and $\left.C_{1}\right)$ satisfy the following $r_{d}(D) \geq r_{d}\left(C_{0}\right)+r_{d}\left(C_{1}\right)$, for all distances d over $\mathrm{Z}_{6}$.

\section{Covering Radius of RePetition CODES}

A q-ary repetition code $\mathrm{C}$ over a finite field $\mathrm{F}_{\mathrm{q}}=\left\{\alpha_{0}=0, \alpha_{1}=1, \alpha_{2}, \alpha_{3}, \ldots, \alpha_{\mathrm{q}-1}\right\}$ is an $[\mathrm{n}, 1, \mathrm{n}]$ code $C=\left\{\bar{\alpha} \mid \alpha \in F_{q}\right\}$, where $\bar{\alpha}=(\alpha, \alpha, \cdots, \alpha)$. The covering radius is $\left\lceil\frac{n(q-1)}{q}\right\rceil$ [9]. Using this, it can be seen easily that the covering radius of block of size $\mathrm{n}$ repetition code [n(q1), $1, \mathrm{n}(\mathrm{q}-1)]$ generated by

$G=[\overbrace{11 \cdots 1}^{n} \overbrace{\alpha_{2} \alpha_{2} \cdots \alpha_{2}}^{n} \overbrace{\alpha_{3} \alpha_{3} \cdots \alpha_{3}}^{n} \cdots \overbrace{\alpha_{q-1} \alpha_{q-1} \cdots}^{n} \overbrace{\alpha_{q-1}}^{n}]$ is $\left\lceil\frac{n(q-1)^{2}}{q}\right\rceil$ since it will be equivalent to a repetition code of length $(q-1)$ n.

Consider the repetition code over $Z_{6}$. There are two types of repetition codes of length $\mathrm{n}$ viz.

1. unit repetition code $C_{\beta}:[\mathrm{n}, 1, \mathrm{n}, \mathrm{n}, \mathrm{n}, \mathrm{n}]$ generated by $G_{\beta}=[\overbrace{11 \cdots 1}^{n}]$.

2. zero repetition code $C_{\alpha}:(\mathrm{n}, 2, \mathrm{n}, 3 \mathrm{n}, 9 \mathrm{n}, 4 \mathrm{n})$ generated by $G_{\beta}=[\overbrace{33 \cdots 3}^{n}]$ and

$$
C_{\gamma}:(\mathrm{n}, 3, \mathrm{n}, 2 \mathrm{n}, 4 \mathrm{n}, 3 \mathrm{n}) \text { generated by } G_{\beta}=[\overbrace{2424 \cdots 24}^{n}] \text { or }[\overbrace{4242 \cdots 42}^{n}] \text {. }
$$

The code generated by $[22 \ldots 2]$ and $[44 \ldots 4]$ are equivalent to the code $C_{\gamma}$.

The following result determines the covering radius with respect to the Lee distance, Euclidean distance and Chinese Euclidean distance. 


\section{Theorem 3. 1.}

$$
\mathrm{r}_{\mathrm{L}}\left(C_{\alpha}\right)=\frac{3 n}{2}, \quad n \leq r_{L}\left(C_{\gamma}\right) \leq \frac{5 n}{3}, \quad r_{L}\left(C_{\beta}\right)=\frac{3 n}{2} .
$$

Proof. By the definition $\mathrm{r}_{\mathrm{L}}\left(C_{\alpha}\right)=\max _{x \in z_{6}^{n}}\left\{\min _{c \in C}\{d(x, c)\}\right\}$. Let $\mathrm{x}=\overbrace{33 \ldots 300 \ldots 0}^{\frac{n}{2}} \overbrace{3 . . .0}^{\frac{n}{2}} \in Z_{6}^{n}$. The code $C_{\alpha}=\left\{\alpha(33 \ldots .3) \mid \alpha \in Z_{6}^{n}\right\}$, that is $C_{\alpha}=\{00 \ldots ., 33 \ldots 3\}$, generated by [3 3 $\left.\ldots .3\right]$ is an (n, 2, n) code.

Then $\mathrm{d}_{\mathrm{L}}(\mathrm{x}, 00 \ldots . .0)=\mathrm{wt}_{\mathrm{L}}(\overbrace{33 \ldots 300 \ldots 0}^{\frac{n}{2}}-00 \ldots .0)=\frac{n}{2} \mathrm{wt}_{\mathrm{L}}(3)=\frac{3 n}{2}$, since the Lee weight of 3 is 3 and $\mathrm{d}_{\mathrm{L}}(\mathrm{x}, 33 \ldots 3)=\frac{3 n}{2}$. Therefore, $\mathrm{d}_{\mathrm{L}}\left(\mathrm{x}, C_{\alpha}\right)=\min \left\{\frac{3 n}{2}, \frac{3 n}{2}\right\}=\frac{3 n}{2}$ and hence $\mathrm{r}_{\mathrm{L}}\left(C_{\alpha}\right) \geq \frac{3 n}{2}$. Let $\mathrm{x}$ be any word in $Z_{6}^{n}$. Let us take $\mathrm{x}$ has $\omega_{0}$ coordinates as 0 's, $\omega_{1}$ coordinates as 1 's, $\omega_{2}$ coordinates as 2 's, $\omega_{3}$ coordinates as 3 's, $\omega_{4}$ coordinates as 4 's and $\omega_{5}$ coordinates as 5 's, then $\omega_{0+} \omega_{1+} \omega_{2+} \omega_{3}$ ${ }_{+} \omega_{4+} \omega_{5}=$ n. Since $C_{\alpha}=\{00 \ldots 0,33 \ldots 3\}$ and Lee weight of 0 is $0,1,5$ is $1,2,4$ is 2 and 3 is 3 , $d_{L}(x, 00 \ldots 0)=n-\omega_{0+} \omega_{2+2} \omega_{3}+\omega_{4}$ and $d_{L}(x, 33 \ldots 3)=n-\omega_{3+2} \omega_{0+} \omega_{1+} \omega_{5}$.

Thus $d_{L}\left(x, C_{\alpha}\right)=\min \left\{\mathrm{n}-\omega_{0+} \omega_{2+2} \omega_{3}+\omega_{4}, \mathrm{n}-\omega_{3+2} \omega_{0+} \omega_{1+} \omega_{5}\right\}$. Since the minimum of $\left\{\mathrm{n}-\omega_{0}\right.$ $\left.{ }_{+} \omega_{2+2} \omega_{3}+\omega_{4}, \mathrm{n}-\omega_{3+2} \omega_{0+} \omega_{1+} \omega_{5}\right\}$ is less than or equal to its average, implies $\mathrm{d}_{\mathrm{L}}\left(\mathrm{x}, C_{\alpha}\right) \leq \mathrm{n}+\frac{n}{2}=$ $\frac{3 n}{2}$ and $\mathrm{r}_{\mathrm{L}}\left(C_{\alpha}\right) \leq \frac{3 n}{2}$. Hence, $\mathrm{r}_{\mathrm{L}}\left(C_{\alpha}\right)=\frac{3 n}{2}$. The correspondent arguments of $\gamma$ type, so, $\mathrm{n} \leq$ $\mathrm{r}_{\mathrm{L}}\left(C_{\alpha}\right) \leq \frac{5 n}{2}$. The covering radius $\mathrm{r}_{\mathrm{L}}\left(C_{\beta}\right) \leq \frac{3 n}{2}$. To show that $\mathrm{r}_{\mathrm{L}}\left(C_{\beta}\right) \geq \frac{3 n}{2}$.

Let $\quad x=\overbrace{00 \cdots 011 \cdots 122 \cdots 2}^{t} \overbrace{23 \cdots 3}^{t} \overbrace{344 \cdots 4}^{t} \overbrace{455 \cdots 5}^{n-5 t} \in Z_{6}^{n}$, where $t=\left\lfloor\frac{n}{6}\right\rfloor$, then $d_{L}(x, 00 \ldots 0)=n+3 t, d_{L}(x, 11 \ldots 1)=2 n-3 t, d_{L}(x, 22 \ldots 2)=3 n-9 t, d_{L}(x, 33 \ldots 3)=2 n-2 t$, $\mathrm{d}_{\mathrm{L}}(\mathrm{x}, 44 \ldots . .4)=\mathrm{n}+3 \mathrm{t}$ and $\mathrm{d}_{\mathrm{L}}(\mathrm{x}, 55 \ldots .5)=9 \mathrm{t}$. Therefore $\mathrm{r}_{\mathrm{L}}\left(C_{\beta}\right) \geq \min \{\mathrm{n}+3 \mathrm{t}, 2 \mathrm{n}-3 \mathrm{t}, 3 \mathrm{n}-9 \mathrm{t}, 9 \mathrm{t}\} \geq \frac{3 n}{2}$ and $\mathrm{r}_{\mathrm{L}}\left(C_{\beta}\right)=\frac{3 n}{2}$.

The above similar arguments can be used to compute the covering radius of Euclidean weight and Chinese Euclidean weight for the $\alpha$ type, $\beta$ type and $\gamma$ type codes over $\mathrm{Z}_{6}$ (Euclidean weight of $Z_{6}$ of 0 is 0,1 and 5 are 1,2 and 4 are 4 and 3 is 9 and Chinese Euclidean weight of $Z_{6}$ of 0 is 0,1 and 5 are 1,2 and 4 are 3 and 3 is 4). We have the following theorem

\section{Theorem 3. 2.}

$$
\mathrm{r}_{\mathrm{E}}\left(C_{\alpha}\right)=\frac{9 n}{2}, \quad 2 n \leq r_{E}\left(C_{\gamma}\right) \leq \frac{11 n}{3} \text { and } r_{E}\left(C_{\beta}\right)=\frac{19 n}{6}
$$




\section{Theorem 3. 3.}

$$
\mathrm{r}_{\mathrm{CE}}\left(C_{\alpha}\right)=2 n, \quad \frac{3 n}{2} \leq r_{C E}\left(C_{\gamma}\right) \leq 2 n \quad \text { and } r_{C E}\left(C_{\beta}\right)=2 n .
$$

In order to determine the covering radius of $\mathrm{Z}_{6}$ two blocks each of size $\mathrm{n}$ repetition code BRep $^{2 n}:[2 n, 1, n, 2 n, 4 n, n]$ generated by $G=[\overbrace{11 \cdots 133 \cdots 3}^{n}]$. We have following theorem

\section{Theorem 3. 4.}

Let $\mathrm{C}$ be a code over $\mathrm{Z}_{6}$ generated by the matrix $G=[\overbrace{11 \cdots 133 \cdots 3}^{n}]$, then $\mathrm{r}_{\mathrm{L}}\left(\mathrm{BRep}^{2 \mathrm{n}}\right)=$ $3 n, r_{E}\left(\left\{B R e p^{2 n}\right)=\frac{46 n}{6}\right.$ and $r_{C E}\left(B \operatorname{Bep}^{2 n}\right)=4 n$.

\section{Proof.}

By Theorem 3.1, the Proposition 2.1 and the given generator matrix G, we get

$$
r_{L}\left(\left\{\text { BRep }^{2 n}\right) \geq 3 n\right.
$$

For the reverse inequality, let $\mathrm{x}=(\mathrm{v} \mid \mathrm{w}) \in Z_{6}^{2 n}$ and let us take in $\mathrm{v}, 0$ appears $\mathrm{r}_{0}$ times, 1 appears $r_{1}$ times, 2 appears $r_{2}$ times, 3 appears $r_{3}$ times, 4 appears $r_{4}$ times and 5 appears $\mathrm{r}_{5}$ times and in w, 0 appears $\mathrm{s}_{0}$ times, 1 appears $\mathrm{s}_{1}$ times, 2 appears $\mathrm{s}_{2}$ times, 3 appears $\mathrm{s}_{3}$ times, 4 appears $\mathrm{s}_{4}$ times and 5 appears $\mathrm{s}_{5}$ times with $\sum_{i=0}^{5} r_{i}=n=\sum_{i=0}^{5} s_{i}$. Then $\mathrm{d}_{\mathrm{L}}\left(\mathrm{x}, \mathrm{c}_{0}\right)=$ $2 n-r_{0}+r_{2}+2 r_{3}+r_{4}-s_{0}+s_{2}+2 s_{3}+s_{4}, \quad d_{L}\left(x, \quad c_{1}\right)=2 n-r_{1}+r_{3}+2 r_{4}+r_{5}-s_{3}+2 s_{0}+s_{1}+s_{5}, d_{L}\left(x, c_{2}\right)=2 n-$ $r_{2}+r_{0}+r_{4}+2 r_{5}-s_{0}+s_{2}+2 s_{3}+s_{4}, \quad d_{L}\left(\quad x, c_{3}\right)=2 n-r_{3}+2 r_{0}+r_{1}+r_{5}-s_{3}+2 s_{0}+s_{1}+s_{5}, \quad d_{L}\left(x, c_{4}\right)=2 n-$ $\mathrm{r}_{4}+\mathrm{r}_{0}+2 \mathrm{r}_{1}+\mathrm{r}_{2}-\mathrm{s}_{0}+\mathrm{s}_{2}+2 \mathrm{~s}_{3}+\mathrm{s}_{4}$ and $\mathrm{d}_{\mathrm{L}}\left(\mathrm{x}, \mathrm{c}_{5}\right)=2 \mathrm{n}-\mathrm{r}_{5}+\mathrm{r}_{1}+2 \mathrm{r}_{2}+\mathrm{r}_{3}-\mathrm{s}_{3}+2 \mathrm{~s}_{0}+\mathrm{s}_{1}+\mathrm{s}_{5}$.

We get

$d_{L}\left(x, B R e p^{2 n}\right)=\min \left\{d_{L}\left(x, c_{0}\right), d_{L}\left(x, c_{1}\right), d_{L}\left(x, c_{2}\right), d_{L}\left(x, c_{3}\right), d_{L}\left(x, c_{4}\right), d_{L}\left(x, c_{5}\right)\right\}$

$$
\begin{gathered}
\leq\left\{2 n-r_{0}+r_{2}+2 r_{3}+r_{4}-s_{0}+s_{2}+2 s_{3}+s_{4}+2 n-r_{1}+r_{3}+2 r_{4}+r_{5}-s_{3}+2 s_{0}+s_{1}+s_{5}+\right. \\
2 n-r_{2}+r_{0}+r_{4}+2 r_{5}-s_{0}+s_{2}+2 s_{3}+s_{4}+2 n-r_{3}+2 r_{0}+r_{1}+r_{5}-s_{3}+2 s_{0}+s_{1}+s_{5}+ \\
\left.2 n-r_{4}+r_{0}+2 r_{1}+r_{2}-s_{0}+s_{2}+2 s_{3}+s_{4}+2 n-r_{5}+r_{1}+2 r_{2}+r_{3}-s_{3}+2 s_{0}+s_{1}+s_{5}\right\} / 6 .
\end{gathered}
$$

Therefore, $\mathrm{d}_{\mathrm{L}}\left(\mathrm{x}, \mathrm{BRep}^{2 \mathrm{n}}\right) \leq 3 n$. Thus $\mathrm{r}_{\mathrm{L}}\left(\left\{\mathrm{BRep}^{2 \mathrm{n}}\right) \leq 3 \mathrm{n}\right.$

By the Equations (3.1) and (3.2), so $r_{L}\left(B \operatorname{Re} p^{2 n}\right)=3 n$.

Similarly, $\mathrm{r}_{\mathrm{E}}\left(\mathrm{BRep}^{2 \mathrm{n}}\right)=\frac{46 n}{6}$ and $\mathrm{r}_{\mathrm{CE}}\left(\mathrm{BRep}^{2 \mathrm{n}}\right)=4 \mathrm{n}$. 
One can also define a $Z_{6}$ codes of three blocks each of size $n$ repetition code BRep ${ }^{3 n}:[3 n$, $1,2 \mathrm{n}, 4 \mathrm{n}, 8 \mathrm{n}, 6 \mathrm{n}]$ generated by $G=[\overbrace{11 \cdots 122 \cdots 233 \cdots 3}^{n}]$. The proof of the theorem 3.5 and 3.6 is similar to the theorem 3. 4, we can state following

\section{Theorem 3. 5.}

Let $\mathrm{C}$ be a code over $\mathrm{Z}_{6}$ generated by the matrix $G=[\overbrace{11 \cdots 122 \cdots 233 \cdots 3}^{n}]$. then

$1.4 \mathrm{n} \leq \mathrm{r}_{\mathrm{L}}\left(\mathrm{BRep}^{3 \mathrm{n}}\right) \leq \frac{9 n}{2}$,

2. $\frac{29 n}{3} \leq \mathrm{r}_{\mathrm{E}}\left(\mathrm{BRep}^{3 \mathrm{n}}\right) \leq \frac{34 n}{3}$ and

3. $\frac{11 n}{2} \leq r_{C E}\left(B \operatorname{Re} p^{3 n}\right) \leq \frac{37 n}{6}$.

In $Z_{6}$, the four blocks each of size $n$ repetition code BRep ${ }^{4 n}$ : [4n, 1, 2n, 6n, 12n, 8n] generated by $G=[\overbrace{11 \cdots 122 \cdots 233 \cdots 344 \cdots 4}^{n}]$. We have following theorem

\section{Theorem 3. 6.}

Let $\mathrm{C}$ be a code over $\mathrm{Z}_{6}$ generated by the matrix $G=[\overbrace{11 \cdots 122 \cdots 233 \cdots 3}^{n} \overbrace{44 \cdots 4}^{n}]$, then 1. $5 \mathrm{n} \leq \mathrm{r}_{\mathrm{L}}\left(\right.$ BRep $\left.^{4 \mathrm{n}}\right) \leq \frac{19 n}{3}$,

2. $\frac{38 n}{3} \leq r_{E}\left(B \operatorname{Re} p^{4 n}\right) \leq \frac{45 n}{3}$ and

3. $7 \mathrm{n} \leq \mathrm{r}_{\mathrm{CE}}\left(\right.$ BRep $\left.^{4 \mathrm{n}}\right) \leq \frac{49 n}{6}$.

In order to determine the covering radius of $\mathrm{Z}_{6}$ codes of the five blocks each of size $\mathrm{n}$ repetition code $\operatorname{BRep}^{5 n}$ : [5n, 1, 3n, 8n, 16n, 12n] generated by

$$
G=[\overbrace{11 \cdots 122 \cdots 233 \cdots 344 \cdots 455 \cdots 5}^{n} \overbrace{2}^{n} \overbrace{}^{n} \overbrace{}^{n}
$$

We have

\section{Theorem 3. 7.}

Let $\mathrm{C}$ be a code generated by the matrix $G=[\overbrace{11 \cdots 122 \cdots 233 \cdots 344 \cdots 455 \cdots 5}^{n}]$.

Then, 
1. $\frac{13 n}{2} \leq r_{L}\left(B \operatorname{Re} p^{5 n}\right) \leq \frac{47 n}{6}$,

2. $\frac{89 n}{6} \leq r_{E}\left(B \operatorname{Re} p^{5 n}\right) \leq \frac{109 n}{6}$ and

3. $9 n \leq r_{C E}\left(\operatorname{BRep}^{5 n}\right) \leq \frac{55 n}{6}$.

The two different length of Block repetition code of size $m$ and $n$ is BRep ${ }^{m+n}:[m+n, 1, m$, $\min \{2 m, m+2 n\}, \min \{4 m, 4 m+6 n\}, \min \{3 m, 3 m+2 n\}]$ generated by

$$
G=[\overbrace{11 \cdots 133 \cdots 3}^{m}] . \text { We have the following theorem }
$$

\section{Theorem 3.8.}

- $\quad \frac{3 m+3 n}{2} \leq \mathrm{r}_{\mathrm{L}}\left(\mathrm{BRep}^{\mathrm{m}+\mathrm{n}}\right) \leq \frac{3 m+4 n}{2}$

- $\mathrm{r}_{\mathrm{E}}\left(\mathrm{BRep}^{\mathrm{m}+\mathrm{n}}\right)=\frac{19 m+27 n}{6}$ and

- $r_{\mathrm{CE}}\left(B R e p^{m+n}\right)=2 m+2 n . \$$

\section{Proof.}

By theorem 3.1 and by the above generator matrix

$$
\mathrm{r}_{\mathrm{L}}\left(\mathrm{BRep}^{\mathrm{m+n}}\right) \geq \frac{3 m}{2}+\frac{3 n}{2}=\frac{3 m+3 n}{2}
$$

Let $\mathrm{z}=(\mathrm{x} \mid \mathrm{y}) \in Z_{6}^{m+n}$ where $\mathrm{x} \in Z_{6}^{m}$ and $\mathrm{y} \in Z_{6}^{n}$. Let us take $\mathrm{x}$ has $\mathrm{m}_{0}$ times 0 as coordinates, $m_{1}$ times 1 as coordinates, $m_{2}$ times 2 as coordinates $m_{3}$ times 3 as coordinates

$\mathrm{m}_{4}$ times 4 as coordinates and $\mathrm{m}_{5}$ times 5 as coordinates and $\mathrm{y}$ has $\mathrm{n}_{0}$ times 0 as coordinates, $\mathrm{n}_{1}$ times 1 as coordinates, $\mathrm{n}_{2}$ times 2 as coordinates $\mathrm{n}_{3}$ times 3 as coordinates $\mathrm{n}_{4}$ times 4 as coordinates and $\mathrm{n}_{5}$ times 5 as coordinates such that $\sum_{i=0}^{5} m_{i}=m$ and $\sum_{i=0}^{5} n_{i}=n$. Then by the above Matrix, the code is $\mathrm{C}=\left\{\mathrm{c}_{0}=(00 \ldots 000 \ldots),. \mathrm{c}_{1}=\left(\begin{array}{lll}11 \ldots .13 & 3\end{array}\right.\right.$

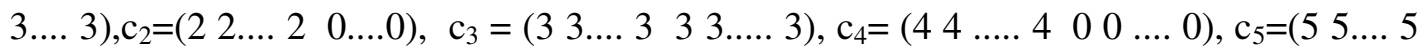
$33 \ldots 3)\}$.

$$
\begin{aligned}
\mathrm{d}_{\mathrm{L}}\left(\mathrm{z}, \mathrm{c}_{0}\right) & =\mathrm{wt}_{\mathrm{L}}\left(\mathrm{z}-\mathrm{c}_{0}\right)=\mathrm{wt}_{\mathrm{L}}\left((\mathrm{x} \mid \mathrm{y})-\mathrm{c}_{0}\right)=\mathrm{wt}_{\mathrm{L}}\left(\mathrm{x}-\mathrm{c}_{0}\right)+\mathrm{wt}_{\mathrm{L}}\left(\mathrm{y}-\mathrm{c}_{0}\right) \\
& =\mathrm{m}_{1}+2 \mathrm{~m}_{2}+3 \mathrm{~m}_{3}+4 \mathrm{~m}_{4}+5 \mathrm{~m}_{5}+\mathrm{n}_{1}+2 \mathrm{n}_{2}+3 \mathrm{n}_{3}+4 \mathrm{n}_{4}+5 \mathrm{n}_{5}, \text { since the }
\end{aligned}
$$

Lee weight of 2,4 is 2 and 3 is 3 and 1,5 is 1 .

Thus $\quad d_{L}\left(z, c_{0}\right)=m+n-m_{0}+m_{2}+2 m_{3}+m_{4}-n_{0}+n_{2}+2 n_{3}+n_{4}$.

Similarly, $d_{L}\left(z, c_{1}\right)=m+n-m_{1}+m_{3}+2 m_{4}+m_{5}-n_{3}+2 n_{0}+n_{1}+2 n_{5}$, $d_{L}\left(z, c_{2}\right)=m+n-m_{2}+m_{0}+1 m_{4}+2 m_{5}-n_{0}+n_{2}+2 n_{3}+n_{4}$, 


$$
\begin{aligned}
& \mathrm{d}_{\mathrm{L}}\left(\mathrm{z}, \mathrm{c}_{3}\right)=\mathrm{m}+\mathrm{n}-\mathrm{m}_{3}+2 \mathrm{~m}_{0}+\mathrm{m}_{1}+2 \mathrm{~m}_{5}-\mathrm{n}_{3}+2 \mathrm{n}_{0}+\mathrm{n}_{1}+2 \mathrm{n}_{5}, \\
& \mathrm{~d}_{\mathrm{L}}\left(\mathrm{z}, \mathrm{c}_{4}\right)=\mathrm{m}+\mathrm{n}-\mathrm{m}_{4}+\mathrm{m}_{0}+2 \mathrm{~m}_{1}+\mathrm{m}_{2}-\mathrm{n}_{0}+\mathrm{n}_{2}+2 \mathrm{n}_{3}+\mathrm{n}_{4} \text { and } \\
& \mathrm{d}_{\mathrm{L}}\left(\mathrm{z}, \mathrm{c}_{5}\right)=\mathrm{m}+\mathrm{n}-\mathrm{m}_{5}+\mathrm{m}_{1}+2 \mathrm{~m}_{2}+\mathrm{m}_{3}-\mathrm{n}_{3}+2 \mathrm{n}_{0}+\mathrm{n}_{1}+2 \mathrm{n}_{5} .
\end{aligned}
$$

Therefore,

$$
\begin{aligned}
& \mathrm{d}_{\mathrm{L}}\left(\mathrm{z}, \text { BRep }^{\mathrm{m}+\mathrm{n}}\right) \leq\left.\left\{\mathrm{d}_{\mathrm{L}}\left(\mathrm{z}, \mathrm{c}_{0}\right)+\mathrm{d}_{\mathrm{L}}\left(\mathrm{z}, \mathrm{c}_{1}\right)+\mathrm{d}_{\mathrm{L}}\left(\mathrm{z}, \mathrm{c}_{2}\right)+\mathrm{d}_{\mathrm{L}}\left(\mathrm{z}, \mathrm{c}_{3}\right)+\mathrm{d}_{\mathrm{L}}\left(\mathrm{z}, \mathrm{c}_{4}\right\}\right)+\mathrm{d}_{\mathrm{L}}\left(\mathrm{z}, \mathrm{c}_{5}\right)\right\} / 6 \\
&=\left\{\mathrm{m}+\mathrm{n}-\mathrm{m}_{0}+\mathrm{m}_{2}+2 \mathrm{~m}_{3}+\mathrm{m}_{4}-\mathrm{n}_{0}+\mathrm{n}_{2}+2 \mathrm{n}_{3}+\mathrm{n}_{4}+\mathrm{m}+\mathrm{n}-\mathrm{m}_{1}+\mathrm{m}_{3}+2 \mathrm{~m}_{4}+\right. \\
& \mathrm{m}_{5-} \mathrm{n}_{3}+2 \mathrm{n}_{0}+\mathrm{n}_{1}+2 \mathrm{n}_{5}+\mathrm{m}+\mathrm{n}-\mathrm{m}_{2}+\mathrm{m}_{0}+1 \mathrm{~m}_{4}+2 \mathrm{~m}_{5}-\mathrm{n}_{0}+\mathrm{n}_{2}+2 \mathrm{n}_{3}+ \\
& \mathrm{n}_{4}+\mathrm{m}+\mathrm{n}-\mathrm{m}_{3}+2 \mathrm{~m}_{0}+\mathrm{m}_{1}+2 \mathrm{~m}_{5}-\mathrm{n}_{3}+2 \mathrm{n}_{0}+\mathrm{n}_{1}+2 \mathrm{n}_{5}+\mathrm{m}+\mathrm{n}-\mathrm{m}_{4}+\mathrm{m}_{0}+ \\
&\left.2 \mathrm{~m}_{1}+\mathrm{m}_{2}-\mathrm{n}_{0}+\mathrm{n}_{2}+2 \mathrm{n}_{3}+\mathrm{n}_{4}+\mathrm{m}+\mathrm{n}-\mathrm{m}_{5}+\mathrm{m}_{1}+2 \mathrm{~m}_{2}+\mathrm{m}_{3}-\mathrm{n}_{3}+2 \mathrm{n}_{0}+\mathrm{n}_{1}+2 \mathrm{n}_{5}\right\} / 6 \\
& \mathrm{~d}_{\mathrm{L}}\left(\mathrm{z}, \text { BRep }^{\mathrm{m}+\mathrm{n}}\right)= \mathrm{m}+\mathrm{n}+\left\{3 \mathrm{~m}+3 \mathrm{n}+3 \mathrm{n}_{5}\right\} / 6 \\
&= \mathrm{m}+\mathrm{n}+3 \mathrm{~m}+3 \mathrm{n}+3 \mathrm{n} / 6, \text { since } \mathrm{n}_{5} \leq \mathrm{n} \\
& \mathrm{d}_{\mathrm{L}}\left(\mathrm{z}, \text { BRep }^{\mathrm{m}+\mathrm{n}}\right)=\{3 \mathrm{~m}+4 \mathrm{n}\} / 2 . \\
&{\text { Thus } \mathrm{r}_{\mathrm{L}}\left(\text { BRep }^{\mathrm{m}+\mathrm{n}}\right) \leq}\{3 \mathrm{~m}+4 \mathrm{n}\} / 2
\end{aligned}
$$

From equation (3.3) and (3.4), $\{3 m+3 n\} / 2 \leq r_{L}\left(\left\{B R e p^{m+n}\right) \leq\{3 m+4 n\} / 2\right.$. Similar arguments of above, we have $r_{E}\left(B \operatorname{Rep}^{\mathrm{m+n}}\right)=\{19 m+27 n\} / 6$ and $r_{C E}\left(B R e p^{m+n}\right)=2 m+2 n$.

In a three different Block repetition code of length is $m, n$ and o is $B R e p^{m+n+o}:[m+n+o$, $1,2 m, \min \{4 m, 2 m+2 n+2 o\}, \min \{8 m, 8 m+4 n+4 o\}, \min \{6 m, 6 m+n+o\}]$ generated by $G=[\overbrace{11 \cdots 122 . .233 \cdots 3}^{m}]$. We have the following theorem

\section{Theorem 3.9.}

- $\{m+2 n+2 o\} / 2 \leq r_{L}\left(B R e p^{m+n+o}\right) \leq\{9 m+10 n+9 o\} / 6$,

- $\{13 m+9 n+12 o\} / 6 \leq r_{E}\left(B_{R e p}{ }^{m+n+o}\right) \leq\{13 m+16 n+15 o\} / 6$ and

- $2 m+\{3 n\} / 2+2 o \leq r_{C E}\left(B R e p^{m+n+o}\right) \leq 2 m+2 n+2 o$.

Four different Block repetition code of length are $m, n, o$ and $p: B R p^{m+n+o+p}:[m+n+o+p$, $1,2 m, \min \{6 m, 2 m+2 n+2 o+2 p\}, \min \{12 m, 12 m+2 n+2 o+2 p\}, \min \{8 m$, $3 m+2 n+2 o+2 p\}]$ generated by

$$
G=[\overbrace{11 \cdots 122 \cdots 233 \cdots 344 \cdots 4}^{m}] \text {. We have the following }
$$

\section{Theorem 3.10.}

- $m+n+o+p \leq r_{L}\left(B_{R e p}^{m+n+o+p}\right) \leq\{9 m+10 n+9 o+10 p\} / 6$,

- $\{13 m+12 n+9 o+12 p\} / 6 \leq r_{E}\left(B_{R e p}{ }^{m+n+o+p}\right) \leq\{13 m+16 n+15 o+16 p\} / 6$ and

- $2 \mathrm{~m}+\{3 \mathrm{n}\} / 2+2 \mathrm{o}+\{3 \mathrm{p}\} / 2 \leq \mathrm{r}_{\mathrm{CE}}\left(\right.$ BRep $\left.^{\mathrm{m}+\mathrm{n}+\mathrm{o}+\mathrm{p}}\right) \leq 2 \mathrm{~m}+\{13 \mathrm{n}\} / 6+2 \mathrm{o}+2 \mathrm{p}$.

The five different Block repetition code of length of size are $\mathrm{m}, \mathrm{n}, \mathrm{o}, \mathrm{p}$ and $\mathrm{q}$, BRep $^{m+n+o+p+q}:[m+n+o+p+q, 1,3 m, \min \{8 m, 2 m+2 n+2 o+2 p+q\}, \min \{16 m, 4 m+4 n+4 o$ $+4 p+3 q\}, \min \{12 m, 3 m+3 n+3 o+2 p+1 q\}]$ generated by 


$$
G=[\overbrace{11 \cdots 122 \cdots 233 \cdots 344 \cdots 455 \cdots 5}^{m}] \text {. We have }
$$

\section{Theorem 3.11.}

- $\{3 m+2 n+3 o+2 p+3 q\} / 2 \leq r_{L}\left(\left\{B R e p^{m+n+o+p+q}\right) \leq\{9 m+10 n+9 o+10 p+9 q\} / 6\right.$,

- $\{19 m+12 n+27 o+12 p+19 q\} / 6 \leq r_{E}\left(B R e p^{m+n+o+p+q}\right) \leq\{19 m+22 n+27 o+22 p+19 q\} / 6$ and

- $\{4 m+3 n+4 o+3 p+4 q\} / 2 \leq r_{C E}\left(B R e p^{m+n+o+p+q}\right) \leq 2 m+2 n+2 o+2 p+2 q$.

\section{ACKNOWLEDGEMENTS}

The first author was supported by a grant (F. No: 4-4/2014-15(MRP-SEM/UGC-SERO, Nov.2014)) for the University Grants Commission, South Eastern Regional office, Hyderabad 500001 .

\section{REFERENCES}

[1] Aoki T., Gaborit P., Harada M., Ozeki M., Sol'e P.: On the covering radius of Z4 codes and their lattices. IEEE Trans. Inform. Theory, vol. 45, no. 6, pp. 2162-2168 (1999).

[2] Bhandari M.C., Gupta M.K., Lal A. K.: On Z4 Simplex codes and their gray images. Applied Algebra, Algebraic Algorithms and Error-Correcting Codes, AAECC-13, Lecture Notes in Computer Science 1719, 170-180 (1999).

[3] Bonnecaze A., Sol'e P., Calderbank A. R.: Quaternary quadratic residue codes and unimodular lattices. IEEE Trans. Inform. Theory, 41, 366-377 (1995).

[4] Bonnecaze A., Sol'e P., Bachoc C., Mourrain B.: Type II codes over Z4. IEEE Trans. Inform. Theory,43, 969-976 (1997).

[5] Bonnecaze A., Udaya P.: Cyclic Codes and Self-Dual Codes over F2 + uF2. IEEE Trans. Inform. Theory, 45(4), 1250-1254 (1999).

[6] Cohen G.D., Karpovsky M. G., Mattson H. F., Schatz J. R.: Covering radius-Survey and recent results. IEEE Trans. Inform. Theory, vol.31, no.3, pp.328-343 (1985).

[7] Cohen C., Lobstein A., Sloane N. J. A.: Further Results on the Covering Radius of Codes, IEEE Trans. Inform. Theory, vol.32, no.5, 1986, pp.680-694, (1997).

[8] Chella Pandian P., Durairajan C.: On the Covering Radius of Codes Over Z4 with Chinese Euclidean Weight. Journal on Information Theory, Vol.4, No.4, October 2015.

[9] Durairajan C.: On Covering Codes and Covering Radius of Some Optimal Codes. Ph.D. Thesis, Department of Mathematics, IIT Kanpur, 1996.

[10] Gupta M.K., Durairajan C.: On the Covering Radius of some Modular Codes. Journal of Advances in Mathematics of Computations, 8(2)\}, 9, 2014.

[11] Gupta M.K., David G. Glynn, Aaron Gulliver T.: On Senary Simplex Codes. Lecture Notes in Computer Science. 